\title{
Statuserhebung von Pflegediensten für außerklinische Beatmung
}

\author{
Survey of Nursing Services with Regard to Mechanical Ventilation at Home
}

Autoren

Institute

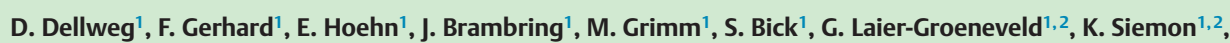
S. Rosseau ${ }^{2}$, W. Windisch ${ }^{2}$

${ }^{1}$ Arbeitsgruppe Pflegedienste der Deutschen interdisziplinären Gesellschaft für außerklinische Beatmung (DIGAB)

${ }^{2}$ Vorstand der Deutschen interdisziplinären Gesellschaft für außerklinische Beatmung (DIGAB) $\begin{array}{lll}\text { eingereicht } & \text { 25. 7. } 2011\end{array}$ akzeptiert nach Revision 15. 8. 2011

\section{Bibliografie}

DOI http://dx.doi.org/ 10.1055/s-0030-1256806

Online-Publikation: 17.10 .2011

Pneumologie 2011; 65: 685-691

(c) Georg Thieme Verlag KG

Stuttgart · New York

ISSN 0934-8387

Korrespondenzadresse

Dr. med. Dominic Dellweg

Kloster Grafschaft

Annostr. 1

57392 Schmallenberg,

d.dellweg@fkkg.de

\section{Zusammenfassung \\ $\nabla$}

Hintergrund: Die ambulante Pflege außerklinisch beatmeter Patienten stellt komplexe Anforderungen an die betreuenden Institutionen. Ebenso komplex sind die Vorgaben der Sozialgesetzgebung, welche die Rahmenbedingungen der Betreuung regeln. Die Deutsche Gesellschaft für Pneumologie und Beatmungsmedizin (DGP) hat zusammen mit der Deutschen Interdisziplinären Gesellschaft für Außerklinische Beatmung (DIGAB) in ihrer Leitlinie hierzu Strukturempfehlungen publiziert und eine Akkreditierung von spezifisch qualifizierten Pflegediensten empfohlen. Rationale: Dieser Akkreditierung vorausgehend führte die Projektgruppe „Pflegedienste“ der DIGAB eine Statuserhebung durch, um die aktuelle Struktur von in der Beatmungspflege tätigen Pflegediensten mit den Empfehlungen der Leitlinie zu vergleichen.

Methode: Selbstauskunft über einen einheitlichen Fragebogen mit insgesamt elf Fragen.

Ergebnis: 37 Pflegedienste mit 78 Niederlassungen, die das gesamte Bundesgebiet betreuen, stellten ihre Fragebögen zur Auswertung zur Verfügung. Während die von der Leitlinie geforderten Ausbildungsstrukturen weitgehend etabliert sind, waren insgesamt nur 43\% der 812 erfassten Patienten an ein Beatmungszentrum oder Weaningzentrum angebunden. 84\% dieser Patienten waren invasiv beatmet.

Obwohl alle Pflegedienste invasiv beatmete Patienten betreuten, fehlten vereinzelt betriebseigene Standards zu spezifischen pflegerischen Maßnahmen.

Schlussfolgerung: Innerhalb dieser Umfrage weisen die Pflegedienste Organisationsformen auf, die in weiten Teilen den Empfehlungen der Leitlinie entsprechen, während die Zentrumsanbindung und damit die ärztliche Versorgung außerklinisch beatmeter Patienten unzureichend ist.

\section{Abstract \\ $\nabla$}

Background: Homecare for mechanically ventilated patients is complex and challenging for homecare institutions. The framework conditions of homecare are regulated by a likewise complex social legislation. The German Respiratory Society (DGP) and the German Interdisciplinary Society for Home Care Ventilation (DIGAB) have published recommendations on the structure of homecare for ventilated patients in their recent guideline and recommended a certification of homecare nursing services.

Rationale: Prior to a certification process, the homecare task force of the DIGAB conducted a survey in order to compare the current structures with the guideline recommendations.

Methods: Voluntary disclosure of information by means of a written questionnaire consisting of eleven items was requested.

Results: 37 homecare institutions with a total of 78 subsidiaries providing service all over Germany returned their questionnaires. While educational standards are mostly in line with the guideline recommendation, it was found that only $43 \%$ of 812 recorded patients followed up with a specialised weaning centre or centre for ventilation. $84 \%$ of these patients were ventilated invasively. In spite of the fact that all homecare institutions took care of invasively ventilated patients, there was a lack of company-owned standards for specific nursing measures.

Conclusions: Homecare for ventilated patients in Germany has reached a decent degree of organisation, while follow-up with specialised centres for ventilation, and with that medical specialist care appears to be underserved. The certification process for homecare institutions should be pursued with emphasis in order to create uniform quality standards. The number of invasively ventilated patients in homecare settings is probably 
Eine Akkreditierung von Beatmungspflegediensten sollte mit Nachdruck verfolgt werden, um bundesweit einheitliche Qualitätsstandards zu schaffen. Die Zahl invasiv beatmeter Patienten im außerklinischen Bereich wurde bisher wahrscheinlich unterschätzt und ist möglicherweise Folge fehlender Weaningkapazitäten.

\section{Einleitung}

$\nabla$

In den siebziger Jahren des letzten Jahrhunderts wurden Probleme in der Fürsorge und Pflege außerklinisch beatmeter Patienten erstmals systematisch erfasst [1].

In der Folge entwickelte man Anforderungsprofile sowie Ausbildungs- und Trainingsprogramme, um eine adäquate Pflege zu gewährleisten [2-4]. In Deutschland lässt die Sozialgesetzgebung (siehe Tab.1) den Verhandlungspartnern (Pflegedienste und Kostenträger) Freiheiten in Bezug auf die Anforderungen und Qualifikationen der Pflegekräfte in der außerklinischen Beatmung. Dies führte zu Unsicherheiten, aber auch regionalen Unterschieden in der Versorgung außerklinisch beatmeter Patienten. Die deutsche Gesellschaft für Pneumologie und Beatmungsmedizin (DGP) hat zusammen mit der Deutschen Interdisziplinären Gesellschaft für Außerklinische Beatmung (DIGAB) in ihrer gemeinsamen Leitlinie zur außerklinischen Beatmung Empfehlungen zur Struktur und Qualifizierung von Pflegediensten formuliert [5]. Hierbei wurden Standards für die Qualifikation der Fachbereichsleitung und der fachpflegerisch tätigen Pflegekräfte empfohlen (siehe Abschnitt 6.4.2. der Leitlinie).

Des Weiteren wurde eine Akkreditierung der Pflegedienste empfohlen.

Einer solchen Akkreditierungsmaßnahme vorausgehend, initiierte eine Arbeitsgruppe der Projektgruppe Pflege der DIGAB eine Ist-Analyse zur Struktur der in der außerklinischen Beatmungspflege tätigen Pflegedienste auf Basis freiwilliger Angaben. Das Ergebnis dieser Umfrage soll helfen, eine mögliche Diskrepanz zwischen aktuellen Strukturen und den Vorgaben der Leitlinie aufzuzeigen.

Tab. 1 Die wesentlichen gesetzlichen Rahmenbedingungen für die Kostenträger und Leistungserbringer.

\begin{tabular}{|c|c|c|}
\hline \multicolumn{3}{|c|}{ für den Bereich der Pflegeversicherungsleistungen } \\
\hline SGB & $\S$ & Inhalt \\
\hline \multirow[t]{3}{*}{$\mathrm{XI}$} & 71 & Qualifikation der Pflegedienstleitung \\
\hline & 75 & $\begin{array}{l}\text { Gestaltung von Rahmen- und Versorgungs- } \\
\text { verträgen }\end{array}$ \\
\hline & $112 \mathrm{ff}$ & Qualitätssicherung \\
\hline \multicolumn{3}{|c|}{ Für den Bereich der häuslichen Krankenpflege } \\
\hline SGB & $\S$ & Inhalt \\
\hline \multirow[t]{2}{*}{$\mathrm{V}$} & 37 & Leistungsbeschreibung \\
\hline & 132a Abs. 2 & $\begin{array}{l}\text { Bedingungen der Leistungserbringung (z. B. } \\
\text { Mindest-Personalausstattung, Qualifikation für } \\
\text { die Erbringung der Behandlungspflegeleistun- } \\
\text { gen, Fortbildungsverpflichtung, Preise etc.) }\end{array}$ \\
\hline
\end{tabular}

\section{Methodik}

Parallel und in Koordination zu den Inhalten der S2-Leitlinie [5] entwarf die Arbeitsgruppe der Projektgruppe Pflege der DIGAB einen Fragebogen zur Erfassung der Struktur und Strukturqualität, der im Herbst 2009 an alle innerhalb der DIGAB gelisteten Pflegedienste und Pflegekräfte versendet wurde. higher than previously estimated and could be the result of a lack of weaning capacity.

Zusätzlich konnte der Fragebogen frei zugänglich von der Homepage der DIGAB heruntergeladen werden (http://www.heimbeatmung.de/weiterbildung.html).

Für die Auswertung wurde der Rücklauf der Fragebögen bis zum 30. 6. 2010 berücksichtigt. Alle vollständig ausgefüllten Fragebögen wurden digital erfasst. Die Auswertung erfolgte mittels SPSS Software Version 17.0, die geografische Darstellung der Daten mit dem Postleitzahlen Diagramm Editor 3.8.

\section{Ergebnisse}

\section{$\nabla$}

37 Fragebögen von ebenso vielen Pflegediensten mit insgesamt 78 Niederlassungen gingen bis zum 30.6.2010 ein und wurden in die Analyse eingeschlossen. Abb. 1 informiert über die regionale Verteilung innerhalb der Bundesrepublik Deutschland. Bezogen auf die Hauptniederlassung haben die Pflegedienste Einsatzgebiete bzw. Einsatzradien, die bis zu $600 \mathrm{~km}$ entfernt sein können (mittel $83 \pm 76 \mathrm{~km}$ ). Hierdurch ermöglichen allein die in dieser Umfrage erhobenen Pflegedienste eine Versorgung des gesamten Bundesgebietes.

Die Pflegedienste hatten im Mittel zwei, im Median eine Niederlassung (Minimum 1, Maximum 11, SD 2,5 siehe Abb.2a). Die Pflegedienste verfügen über eine durchschnittliche Erfahrung in der Beatmungspflege von 86 Monaten (Minimum 6, Maximum 240 Monate, Median 72 Monate, SD 58 Monate siehe Abb.2 b). Pro Pflegedienst werden im Mittel 22 Patienten betreut (Minimum 3, Maximum 100, Median 12, SD 26 Patienten siehe $\bullet$ Abb. 2c), insgesamt gibt diese Erhebung somit Auskunft über Daten von 812 Patienten.

Hiervon werden $84 \%$ invasiv beatmet. Die Anzahl der pro Pflegedienst betreuten Patienten scheint dabei keinen Einfluss auf die Verteilung zwischen invasivem und nicht-invasivem Beatmungszugang zu haben (R-Quadrat=0,001).

Die Verteilung der Altersklassen in Kleinkinder (bis 6 Jahre), Jugendliche ( 6 bis 17 Jahre) und Erwachsene (volljährig) findet sich in Abb. 3 .

Nach Angaben in den Fragebögen sind dabei $43 \%( \pm 36 \%)$ an ein Beatmungszentrum oder Weaningzentrum angebunden. - Tab. 2 zeigt, wie viel Prozent der Pflegedienste Patienten mit entsprechender Grunderkrankung betreuen.

\section{Qualifikation Fachaufsicht}

Die 37 Pflegedienste gaben für ihre 78 Niederlassungen an, dass die verantwortlichen Fachaufsichten üblicherweise die in - Tab.3 aufgeführten fachlichen Qualifikationen aufweisen. Alle Pflegedienste gaben dabei mindestens eine fachliche Qualifikation an.

\section{Zusatzqualifikation Fachaufsicht}

78\% der Pflegedienste haben Fachaufsichtskräfte mit der Zusatzqualifikation Fachgesundheits- und Krankenpfleger für Anästhesie und Intensivpflege, $87 \%$ haben Aufsichtskräfte mit Beatmungserfahrung (76\% mit klinischer Beatmungserfahrung, 87\% mit außerklinischer Erfahrung), die Länge der Berufserfahrung 


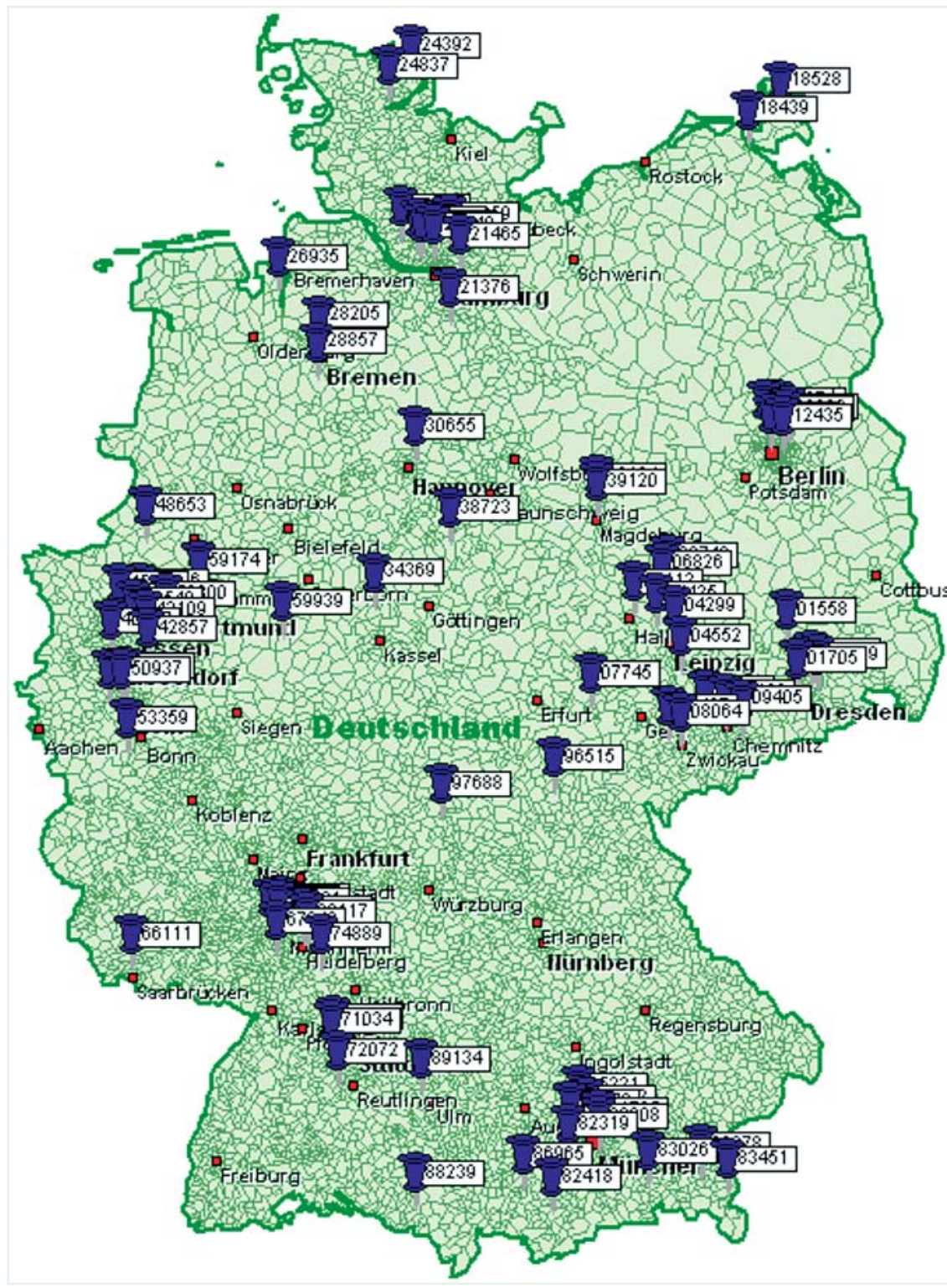

Abb. 1 Verteilung der 78 Niederlassungen auf dem Gebiet der Bundesrepublik Deutschland.

im Beatmungsbereich liegt dabei im Mittel bei 52 Monaten (Minimum 2, Maximum 156, SD 34 Monate). Atmungstherapeuten werden bei $16 \%$ aller befragten Pflegedienste im Bereich Fachaufsicht eingesetzt. Lediglich ein Pflegedienst konnte keine Zusatzqualifikationen für den Bereich Fachaufsicht angeben.

\section{Qualifikation Betreuungspersonal}

- Tab.4 enthält Angaben über die übliche Qualifikation des fachpflegerischen Personals.

\section{Zusatzqualifikation Betreuungspersonal}

76\% der Pflegedienste haben Betreuungspersonal mit der Zusatzqualifikation Fachgesundheits- und Krankenpfleger für Anästhesie und Intensivpflege, 95\% haben Betreuungspersonal mit Beatmungserfahrung (84\% mit klinischer Beatmungserfahrung, 92\% mit außerklinischer Erfahrung). Atmungstherapeuten werden bei $19 \%$ aller befragten Pflegedienste im Bereich Betreuung eingesetzt. Lediglich ein Pflegedienst konnte keine Zusatzqualifikationen für sein Betreuungspersonal angeben.

\section{Andere Qualifikationsformen im Bereich}

Betreuungspersonal

Neben den oben angegebenen Qualifikationsformen arbeiten 22\% der Pflegedienste mit angelernten Laienkräften, 27\% mit einjährig examinierten Pflegekräften, 19\% mit einjährig examinierten Altenpflegekräften und 24\% mit Mitarbeitern, die eine anderweitig nicht näher spezifizierte Qualifikationsform haben.

\section{Weitere Dienstleistungen}

Eine Beratung in sozial-rechtlichen Fragen wird von 78\% und ein Konfliktmanagement von $62 \%$ der Pflegedienste angeboten. Ein Teil der Pflegedienste (40\%) bietet darüber hinaus weitere Dienstleistungen an wie z.B. die Beratung und Organisation der außerklinischen Versorgungslogistik sowie Schulungs- und Rehabilitationsangebote. 

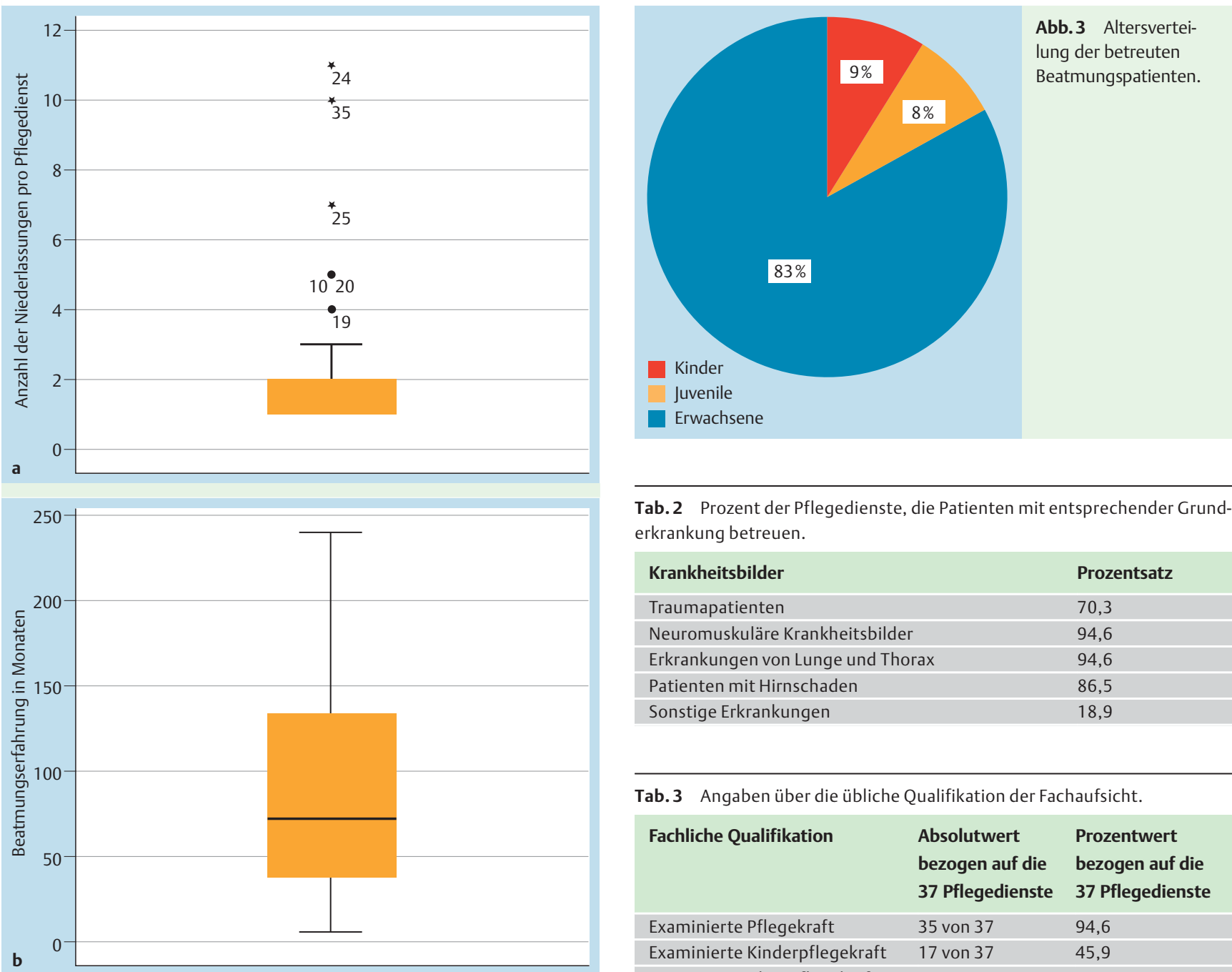

Tab. 2 Prozent der Pflegedienste, die Patienten mit entsprechender Grunderkrankung betreuen.

\begin{tabular}{|l|l|}
\hline Krankheitsbilder & Prozentsatz \\
\hline Traumapatienten & 70,3 \\
\hline Neuromuskuläre Krankheitsbilder & 94,6 \\
\hline Erkrankungen von Lunge und Thorax & 94,6 \\
\hline Patienten mit Hirnschaden & 86,5 \\
\hline Sonstige Erkrankungen & 18,9 \\
\hline
\end{tabular}

Tab.3 Angaben über die übliche Qualifikation der Fachaufsicht.

\begin{tabular}{|l|l|l|}
\hline Fachliche Qualifikation & $\begin{array}{l}\text { Absolutwert } \\
\text { bezogen auf die } \\
\text { 37 Pflegedienste }\end{array}$ & $\begin{array}{l}\text { Prozentwert } \\
\text { bezogen auf die }\end{array}$ \\
\hline & 37 Pflegedienste \\
\hline Examinierte Pflegekraft & 35 von 37 & 94,6 \\
\hline Examinierte Kinderpflegekraft & 17 von 37 & 45,9 \\
\hline Examinierte Altenpflegekraft & 9 von 37 & 24,3 \\
\hline
\end{tabular}

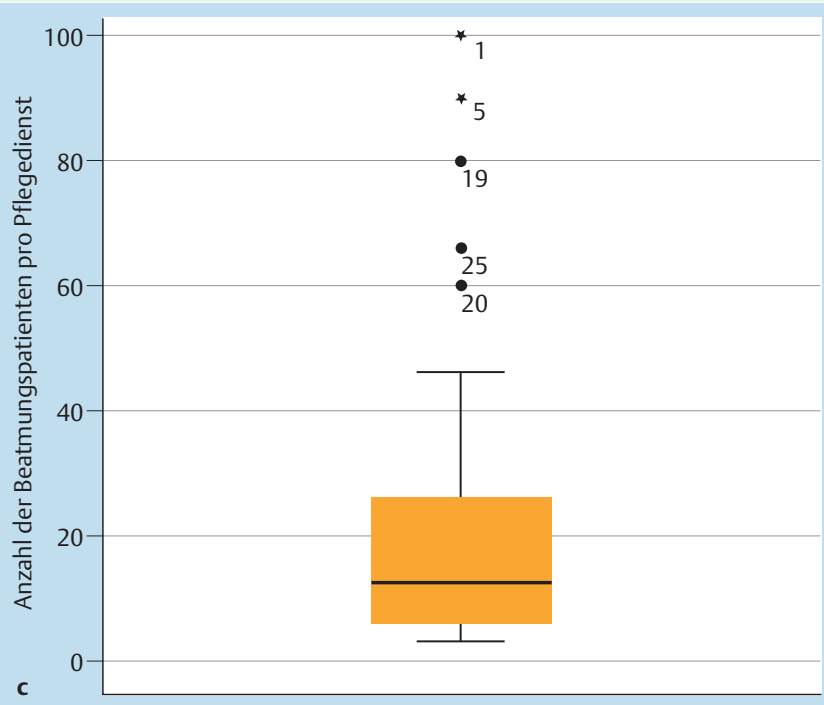

Abb.2 Anzahl der Niederlassungen pro Pflegedienst (a), Zeitraum in Monaten, seit dem der jeweilige Pflegedienst beatmete Patienten betreut (b), Anzahl der betreuten Patienten pro Pflegedienst (c).

Tab.4 Angaben über die übliche Qualifikation des fachpflegerischen Personals.

\begin{tabular}{|l|l|l|}
\hline Ausbildung & $\begin{array}{l}\text { Absolutwert } \\
\text { bezogen auf die } \\
\text { 37 Pflegedienste }\end{array}$ & $\begin{array}{l}\text { Prozentwert } \\
\text { bezogen auf die }\end{array}$ \\
\hline 37 Pflegedienste
\end{tabular}

\section{Qualitätssicherung}

Über die Anwendung bestimmter Standards in der Pflege beatmeter Patienten informiert $\bullet$ Abb.4. Alle untersuchten Pflegedienste führen eine Beatmungsdokumentation und Dienstübergaben durch, bieten Beatmungsfortbildungen an, und haben feste Einarbeitungskonzepte für ihre Mitarbeiter. Fast alle (97\%) führen regelmäßige Mitarbeitergespräche und Teambesprechungen durch und arbeiten mit regelmäßigen Pflegevisiten. Darüber hinaus haben 86\% der Pflegedienste eigene Qualitätszirkel und $50 \%$ bieten eine Supervision an. 


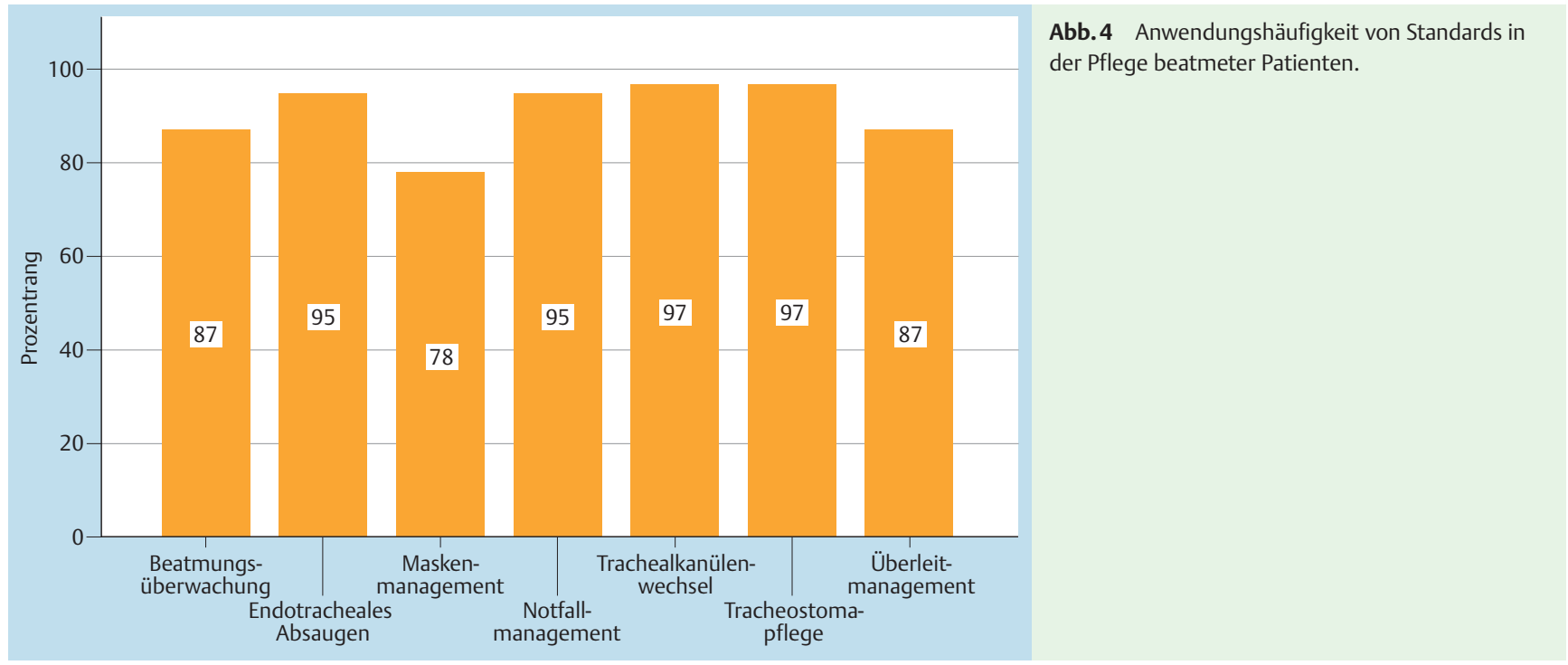

\section{Diskussion}

Parallel zum gewählten Fragebogenverteiler, der möglicherweise mehrere Mitarbeiter des gleichen Pflegedienstes enthielt, konnte der Fragebogen online von der Homepage der DIGAB heruntergeladen werden. Aus diesen Gründen kann bei dieser Umfrage keine Rücklaufquote berechnet werden. Die Eurovent-Studie [6] schätzt die Prävalenz außerklinisch beatmeter Patienten auf 6,6 pro 100000 Einwohner. Das macht bei einer Gesamtbevölkerung in Deutschland von 82000000 [7] eine Patientenzahl von etwa 5412 aus. In der Eurovent-Studie waren insgesamt 13\% der Patienten invasiv beatmet, von daher wäre für die Bundesrepublik eine Zahl von 702 außerklinisch invasiv beatmeter Patienten anzunehmen. Die 812 in dieser Erhebung genannten Patienten ergeben somit insgesamt nur ca. $15 \%$ der in der Eurovent-Studie angenommenen Gesamtfälle, da in der vorliegenden Erhebung aber $84 \%$ der Patienten invasiv beatmet war, ergibt sich hier eine Zahl von 682 invasiv beatmeter Patienten, was ziemlich genau der geschätzten Gesamtzahl aus der Eurovent-Studie entspricht. Die hohe Kongruenz der Zahlen invasiv beatmeter Patienten bei jedoch deutlicher Diskrepanz der Gesamtzahl aller beatmeten Patienten lässt vermuten, dass die Eurovent-Studie die Prävalenz invasiv beatmeter Patienten erheblich unterschätzt.

Wir müssen bei der Diskrepanz der Zahlen auf der anderen Seite bei unserer Umfrage davon ausgehen, dass die in die Auswertung eingeschlossenen 37 Pflegedienste mit ihren insgesamt 78 Niederlassungen nur eine Teilmenge der in der Bundesrepublik tätigen Pflegedienste in der Beatmungspflege darstellen.

Trotzdem zeigt sich hier bereits, dass eine Abdeckung des gesamten Bundesgebietes erreicht wird. Die einzelnen Niederlassungen konzentrieren sich dabei erwartungsgemäß in den Ballungsgebieten. Die Anzahl der Niederlassungen pro Pflegedienst ist nicht homogen verteilt. Liegt der Mittelwert hier bei durchschnittlich zwei Niederlassungen, so gibt es einige wenige Pflegedienste, die eine wesentlich höhere Anzahl an Niederlassungen haben. Neben vielen regional tätigen Pflegediensten sieht man jedoch auch Unternehmen, die bundesweit tätig sind. Hierbei kommt sicher zum Tragen, dass für die Pflegeübernahme in der Heimatregion des Patienten häufig neue, individuelle Pflegeteams zusammengestellt werden. Alle Pflegedienste betreuten sowohl invasiv als auch nicht-invasiv beatmete Patienten, in der Regel lag der Anteil invasiv beatmeter Patienten dabei deutlich über
50\%. In Europa liegt der Anteil der außerklinisch invasiv beatmeten Patienten an der Gesamtzahl aller außerklinisch beatmeten Patienten bei $13 \%$, in Deutschland sogar eher unter 10\% [6]. Diese Diskrepanz scheint plausibel, da viele Patienten mit nicht-invasiver Beatmung die Handhabung der Ventilation selber übernehmen und daher keine spezielle Beatmungspflege in Anspruch nehmen.

Mehr als 80\% der versorgten Patienten sind im Erwachsenenalter; dieser Wert erscheint plausibel und wird aufgrund der Altersstruktur unseres Landes bei gleicher Indikationsstellung der Beatmung sicher eher zunehmen.

Da alle Pflegedienste überwiegend invasiv beatmete Patienten betreuen, wäre zu erwarten gewesen, dass beatmungsspezifische Pflegemaßnahmen, wie die Beatmungsüberwachung, das endotracheale Absaugen oder ein Trachealkanülenmanagement auch in allen Fällen angeboten wird. Dies ist gemäß $\bullet$ Abb. 4 jedoch nicht immer gegeben, was die Frage nach einer weiteren Qualitätskontrolle verstärkt.

Bei der Abfrage der Qualifikationen für die Fachbereiche und Betreuungspflege wurde lediglich erfragt, welche Ausbildungsformen in den einzelnen Betrieben vorkommen. Es kann daher keine Aussage darüber getroffen werden, ob alle eingesetzten Kräfte die in der Leitlinie [5] geforderten Basis- und Zusatzqualifikationen aufweisen. Lediglich ein Pflegedienst gab an, generell keine Zusatzqualifikationen vorweisen zu können. Insgesamt scheint das Berufsbild des Atmungstherapeuten [8] im Bereich der Fachaufsicht mit 16\% und bei der Betreuungspflege mit 19\% der Pflegedienste noch unterrepräsentiert zu sein, was sicherlich an der späten Einführung dieser Ausbildung in Deutschland, verglichen mit anderen Staaten, liegt [9]. Etwa 25\% aller Pflegedienste gaben an, Betreuungspersonal mit geringerer Qualifikation (Laienkräfte, einjährig examinierte Kräfte) in der Versorgung beatmeter Patienten einzusetzen, wobei nicht abgefragt wurde, ob diese Tätigkeit eigenverantwortlich oder unter Aufsicht erfolgt. Die erforderlichen Qualifikationen für die ambulante Grund- und Behandlungspflege regeln im Einzelnen die nach §132a SGB V und SGB XI geschlossenen Verträge zwischen den Pflegediensten und den Kassen. Die aktuelle Leitlinie [5] empfiehlt hier ein einheitliches Vorgehen mit definierten Standards, um die Qualität der Pflege in der außerklinischen Beatmung sicherzustellen.

Die Versorgung außerklinisch beatmeter Patienten stellt häufig eine erhebliche psychologische und häufig auch finanzielle Be- 
lastung für den Patienten und die Familienangehörigen dar [10]. Den Stellenwert einer umfassenden Beratung in sozialrechtlichen Fragen sowie eines Konfliktmanagements wurde von den Pflegediensten erkannt und in den meisten Fällen umgesetzt.

Interne Maßnahmen zur Qualitätssicherung werden nach Angaben der Pflegedienste mit Ausnahme der Supervision in vielfältiger Weise genutzt.

Besorgniserregend erscheint jedoch die Tatsache, dass entgegen der Empfehlungen [5] lediglich 43\% der Patienten an ein Beatmungszentrum oder ein Weaningzentrum angeschlossen sind. Die S2-Leitlinie weist in Kapitel 6 bereits auf die Schwierigkeiten der außerklinischen ärztlichen und fachärztlichen Betreuung beatmeter Patienten hin [5]. Die Beatmung der chronischen respiratorischen Insuffizienz ist in der Regel kein curricularer Bestandteil des Medizinstudiums.

Kenntnisse in diesem Gebiet können daher nicht regelhaft vorausgesetzt werden und sind abhängig von der weiteren individuellen ärztlichen Aus- und Weiterbildung.

Gleichzeitig besteht in Deutschland, zumindest budgetär und anders als in anderen Ländern, eine relativ strikte Trennung von stationärer und ambulanter Medizin.

Letztere ist zudem nochmals in eine hausärztliche Grundversorgung und eine fachärztliche Versorgung der Patienten gegliedert. Während die in der hausärztlich ambulanten Versorgung tätigen Ärzte in der Regel nicht über ausreichende Kenntnisse und technische Überwachungsmöglichkeiten in der Beatmungsmedizin verfügen, bestehen für die fachärztlichen, niedergelassenen und stationär tätigen Ärzte keine Strukturen, um entsprechende Leistungen zu erbringen und abzurechnen.

Der Bedarf an qualifizierter Betreuung vor Ort ist seit langem bekannt und durch Studien belegt. Chatwin et al. konnten zeigen, dass außerklinisch beatmete Patienten im Mittel fast alle zwei Monate aufgrund von medizinischen oder technischen Problemen telefonischen Kontakt zum Beatmungszentrum aufnahmen [11]. In fast $10 \%$ der Fälle verbarg sich hinter der Annahme eines Gerätedefektes durch den Patienten in Wirklichkeit eine klinische Verschlechterung, die ambulant oder stationär behandelt werden musste [11]. Srinivasan et al. beobachtete in einer Kohorte von 150 beatmeten Patienten (76\% davon invasiv) innerhalb eines Jahres insgesamt 189 Kontaktaufnahmen zu den Geräteprovidern wegen technischer Probleme [12]. Ein wirklicher Defekt konnte aber nur in 39\% der Fälle gefunden werden. Farré et al. zeigten in ihren Daten, die parallel zur Eurovent-Studie erhoben wurden, dass die Nachsorge außerklinisch beatmeter Patienten in Europa sehr heterogen organisiert ist [13]. Auch für Deutschland konnte gezeigt werden, dass es keine einheitliche Regelung bei Stör- und Problemfällen und bei Beatmungsproblemen gibt. Mögliche Ansprechpartner in Problemfällen sind die Pflegedienste, niedergelassene oder auch stationär tätige Ärzte sowie die Geräteprovider und deren Außendienstmitarbeiter [13]. Es gibt weder eine einheitliche Verfahrensanleitung, wie im Krisenfall vorzugehen ist, noch gibt es einen gesicherten Informationsaustausch zwischen den genannten Parteien. Vor diesem Hintergrund wird der Ruf nach einem strukturierten Informationsaustausch oder einem Register für Beatmungspatienten verständlich.

Die Tatsache, dass lediglich $43 \%$ der Patienten an ein Beatmungsoder Weaningzentrum angebunden sind, verdeutlicht den akuten Handlungsbedarf in der außerklinischen Beatmungsmedizin. Im Vergleich zur invasiven Beatmung ist die nicht-invasive Beatmung mit einer geringeren Morbidität und Mortalität sowie geringeren Kosten verbunden. Daher stellt sich die Frage, welcher
Anteil der außerklinisch invasiv beatmeten Patienten einen stationären Weaningversuch durchlaufen haben. Gleichzeitig haben die Weaningzentren in Deutschland z.T. erhebliche Wartelisten. Die schlechte Anbindung der Patienten könnte damit auch durch erhebliche Unterkapazitäten in den Zentren hervorgerufen werden. Wie bereits dargestellt, scheint die wirkliche Zahl der invasiv außerklinisch beatmeten Patienten deutlich oberhalb der Zahlenschätzungen der Eurovent-Studie zu liegen. Dies kann bedeuten, dass wir im Vergleich zu den anderen in der EuroventStudie aufgeführten europäischen Ländern eine höhere Quote an invasiv beatmeten Patienten in Deutschland haben. Als Ursache hierfür kommt entweder eine andere Indikationsstellung für die invasive Beatmung oder aber auch eine fehlende Kapazität an Weaningplätzen in Frage.

Des Weiteren zeigt die geringe Anbindungsquote von $43 \%$, dass die vor Ort tätigen Pflegedienste in der Regel keinen beatmungserfahrenen ärztlichen Ansprechpartner haben, den sie in Problemfällen konsultieren können. Es ist daher dringend erforderlich Strukturen zu schaffen, die diese Versorgungslücke füllen. Es gilt Modelle zu entwickeln, die es erlauben, außerklinisch beatmeten Patienten Zugang zu einer im Hinblick auf die Beatmung kompetenten ambulanten ärztlichen Betreuung zu erhalten. Hierzu ist die Bereitschaft von stationär in Beatmungszentren tätigen Ärzten sowie von Fachärzten im niedergelassenen Bereich erforderlich, die dann Verantwortung in der ambulanten Betreuung beatmeter Patienten übernehmen. Gleichzeitig ist die Politik und sind Kostenträger sowie kassenärztliche Vereinigungen aufgerufen, entsprechende Versorgungsmodelle zu strukturieren und zu fördern.

Speziell geschulte Pflegekräfte und Atmungstherapeuten sowie telemedizinische Überwachungssysteme werden in anderen Ländern bereits erfolgreich in der Versorgung außerklinisch beatmeter Patienten eingesetzt [14]. Diese Möglichkeiten sollten bei der Entwicklung eines Versorgungssystems in Deutschland nicht unberücksichtigt bleiben.

\section{Limitationen dieser Umfrage \\ $\nabla$}

Bei den eingegangenen Daten handelt es sich um retrospektive Selbsteinschätzungen freiwillig teilnehmender Pflegedienste bzw. deren Mitarbeiter. Bei Selbsteinschätzungen besteht generell immer die Gefahr, dass positive Attribute über- und negative Attribute unterbewertet werden [15,16]. Diese Gefahr scheint sich zu verstärken, je weiter diese Attribute sich von der Norm entfernen [17]. Wir können daher nicht mit Sicherheit ausschließen, dass die hier dargestellten Daten in entsprechender Weise von der Realität abweichen. Gleichzeitig können wir keine Angaben über die tatsächliche Gesamtzahl der in der Bundesrepublik im Bereich außerklinischer Beatmung tätigen Pflegedienste machen. Wir müssen auf Grund der Freiwilligkeit der Teilnahme von einem Selektionsbias ausgehen, wobei anzunehmen ist, dass Pflegedienste, die die abgefragten Strukturmerkmale weniger gut erfüllen, eher von der Teilnahme dieser Befragung abgesehen haben. 


\section{Zusammenfassung}

Die Ergebnisse dieser auf freiwilligen Angaben beruhender Umfrage zeigt eine Konzentration von Beatmungspflegediensten in Ballungsgebieten. Von hier aus bieten die Pflegedienste dieser Umfrage aber eine bundesweite Versorgung an.

Der Prozentsatz der Patienten, die an ein Beatmungszentrum angeschlossen sind, ist mit $43 \%$ alarmierend gering und verbesserungsbedürftig. Die fachärztlichen Versorgungsstrukturen außerklinisch beatmeter Patienten sind defizitär. Hier gilt es mit neuen Modellen diese Versorgungslücke zu schließen. Die Pflegedienste können für ihr Personal im Bereich Fachaufsicht und Behandlungspflege bereits hohe Standards nachweisen, inwieweit dies auf die Breite der Mitarbeiter zutrifft, kann jedoch nur durch eine detailliertere Abfrage oder im Rahmen einer Akkreditierung geklärt werden. Besonders im Bereich betriebseigener Standards ( $\bullet$ Abb.4) werden nicht alle Maßnahmen in standardisierter Form vorgehalten, die für eine sichere und umfassende Betreuung beatmeter Patienten sinnvoll erscheinen.

Die angestrebte Akkreditierung erscheint daher sinnvoll und sollte baldmöglichst umgesetzt werden. Auch bisher nicht erfasste Pflegedienste sollten dabei jederzeit die Möglichkeit haben, an der geplanten Akkreditierung teilzunehmen. Wahrscheinlich liegt die Anzahl außerklinisch invasiv beatmeter Patienten über der Anzahl bisheriger Schätzungen. Hier ist die Erhebung weiterer Daten dringend indiziert, vor allem um den zusätzlichen Bedarf an Weaningeinheiten besser definieren zu können.

\section{Interessenkonflikt}

Alle Autoren arbeiten in der pflegerischen oder ärztlichen Versorgung beatmeter Patienten.

\section{Literatur}

1 Fischer DA, Prentice WS. Feasibility of home care for certain respiratory-dependent restrictive or obstructive lung disease patients. Chest 1982; 82: 739-743
2 Dougherty JM, Parrish JM, Parra M et al. Part 2: using a competency-based curriculum to train experienced nurses in ventilator care. Pediatr Nurs 1996; 22: $47-50$

3 Dougherty JM, Parrish JM, Hock-Long L. Developing a competency-based curriculum for tracheostomy and ventilator care. Pediatr Nurs 1995; $21: 581-584$

4 Grimm M. [Qualification of nursing staff for home ventilation]. Pneumologie 1999; 53: S126-127

5 Windisch W, Walterspacher S, Siemon Ket al. Guidelines for non-invasive and invasive mechanical ventilation for treatment of chronic respiratory failure. Pneumologie 2010; 64: 640-652

6 Lloyd-Owen SJ, Donaldson GC, Ambrosino N et al. Patterns of home mechanical ventilation use in Europe: results from the Eurovent survey. Eur Respir J 2005; 25: 1025-1031

7 StatistischesBundesamt.Wiesbaden:http://www.destatis.de/jetspeed/ portal/cms/Sites/destatis/Internet/DE/Navigation/Statistiken/Bevoelkerung/Bevoelkerung.psml2011

$8 \mathrm{Karg} O$, Bubulj C, Esche B et al. [The respiratory therapist]. Pneumologie 2008; 62: 685-689

9 Karg $O$, Bonnet $R$, Magnussen $\mathrm{H}$ et al. [Respiratory therapist: introduction of a new profession]. Pneumologie 2004; 58: $854-857$

10 Van Pelt DC, Milbrandt EB, Qin L et al. Informal caregiver burden among survivors of prolonged mechanical ventilation. Am J Respir Crit Care Med 2007; 175: $167-173$

11 Chatwin M, Heather S, Hanak A et al. Analysis of home support and ventilator malfunction in 1,211 ventilator-dependent patients. Eur Respir J 2010; 35: 310-316

12 Srinivasan S, Doty SM, White TR et al. Frequency, causes, and outcome of home ventilator failure. Chest 1998; 114: $1363-1367$

13 Farre R, Lloyd-Owen SJ, Ambrosino $N$ et al. Quality control of equipment in home mechanical ventilation: a European survey. Eur Respir J 2005; 26: $86-94$

14 Vitacca M, Paneroni M, Trainini D et al. At home and on demand mechanical cough assistance program for patients with amyotrophic lateral sclerosis. Am J Phys Med Rehabil 2010; 89: 401 - 406

15 Landsberg B, Bastian I, Plachta-Danielzik $S$ et al. [Self-reported and measured height and weight in adolescents]. Gesundheitswesen 2011; 73: $40-45$

16 Landeck E, Zuhrt R. [Self evaluation and objective dental findings]. Stomatol DDR 1975; 25: 630-635

17 Buchowski MS, Townsend KM, Chen KY et al. Energy expenditure determined by self-reported physical activity is related to body fatness. Obes Res 1999; 7: $23-33$ 